\title{
The novel squamosamide derivative FLZ enhances BDNF/TrkB/CREB signaling and inhibits neuronal apoptosis in APP/PS1 mice
}

\author{
Ning LI, Geng-tao LIU* \\ Department of Pharmacology, Institute of Materia Medica, Peking Union Medical College and Chinese Academy of Medical Sciences, \\ Beijing 100050, China
}

\begin{abstract}
Aim: The aim of this study was to study the effects of compound FLZ, a novel cyclic derivative of squamosamide from Annona glabra, on brain-derived neurotrophic factor (BDNF)/tropomyosin receptor kinase B (TrkB)/cAMP response element-binding protein (CREB) signaling and neuronal apoptosis in the hippocampus of the amyloid precursor protein (APP)/presenilin-1 (PS1) double transgenic mice. Methods: APP/PS1 mice at the age of 5 months and age-matched wild-type mice (WT) were intragastrically administered FLZ (150 $\mathrm{mg} / \mathrm{kg}$ ) or vehicle [0.05\% carboxymethyl cellulose sodium (CMC-Na)] daily for 20 weeks. The levels of BDNF in the hippocampus of WT and APP/PS1 mice were then measured by immunohistochemistry and Western blot analysis. Neuronal apoptosis in mouse hippocampus was detected by Nissl staining. Expression of NGF, NT3, pTrkB (Tyr515)/TrkB, pAkt (Ser473)/Akt, pERK/ERK, pCREB (Ser133)/ CREB, Bcl-2/Bax, and active caspase-3 fragment/caspase-3 in the hippocampus of WT and APP/PS1 mice was detected by Western blot analysis.

Results: Compared with vehicle-treated APP/PS1 mice, FLZ (150 mg/kg) significantly increased BDNF and NT3 expression in the hippocampus of APP/PS1 mice. In addition, FLZ promoted BDNF high-affinity receptor TrkB phosphorylation and activated its downstream ERK, thus increasing phosphorylation of CREB at Ser133 in the hippocampus of APP/PS1 mice. Moreover, FLZ showed neuroprotective effects on neuronal apoptosis by increasing the Bcl-2/Bax ratio and decreasing the active caspase-3 fragment/caspase-3 ratio in the hippocampus of APP/PS1 mice.

Conclusion: FLZ exerted neuroprotection at least partly through enhancing the BDNF/TrkB/CREB pathway and inhibiting neuronal apoptosis in APP/PS1 mice, which suggests that FLZ can be explored as a potential therapeutic agent in long-term Alzheimer's disease therapy.
\end{abstract}

Keywords: FLZ; brain-derived neurotrophic factor; TrkB receptor; phospho-CREB-binding protein; Bcl-2; apoptosis

Acta Pharmacologica Sinica (2010) 31: 265-272; doi: 10.1038/aps.2010.3; published online 15 February 2010

\section{Introduction}

Alzheimer's disease (AD), an age-related neurodegenerative disorder, is characterized by progressive neuronal loss and cognitive impairment. Two pathological features characterize AD besides amyloid plaques and neurofibrillary tangles. One feature is the selective loss of neurons, including basal forebrain cholinergic neurons and neurons in the cortex, hippocampus and certain subcortical regions. This neuronal loss contributes to progressive cognitive deficits ${ }^{[1-3]}$. Another feature is synaptic loss, including decreased neuronal plasticity ${ }^{[4]}$, probably attributable to the aberrant increase of $\beta$-amyloid $(A \beta)$ deposits in the brain ${ }^{[5,6]}$. The pathogenesis of $A D$ remains

\footnotetext{
* To whom correspondence should be addressed.

E-mail liugt@imm.ac.cn

Received 2009-10-26 Accepted 2010-01-07
}

unclear, and the available treatments are somewhat limited. All current treatments approved by Food and Drug Administration (FDA) for AD only have moderate efficacy in slowing the rate of cognitive decline in patients and do not halt progression of the disease ${ }^{[7]}$.

Neurotrophins (NTs), including nerve growth factor (NGF), brain-derived neurotrophic factor (BDNF), neurotrophin 3 (NT3), and NT4/5, which are critical molecules supporting the development, differentiation, maintenance and plasticity of brain function throughout life ${ }^{[8]}$, have been implicated in the pathophysiology of neurodegenerative and psychiatric disorders. Several studies reported that neurotrophic starvation, including NGF or BDNF deficiency, begins in the early stage of $\mathrm{AD}$ and eventually causes neuronal degeneration, cell death and loss of cholinergic neurotransmission in the late stage of $\mathrm{AD}^{[9,10]}$. Neuronal plasticity is directly affected by the levels 
of several neurotrophins, especially BDNF, and evidence has proven that expression of BDNF is impaired in AD patients and AD-like animal models ${ }^{[11]}$. Exogenous addition of BDNF can rescue neurons from death by preventing $A \beta$-induced neurodegeneration in vitro and in vivo ${ }^{[12]}$. BDNF exerts its promoting effect mainly by binding to tropomyosin receptor kinase B (TrkB), leading to TrkB autophosphorylation of tyrosine residues and activation of signaling molecules, including the mitogen-activated protein kinases (MAPK)/extracellular signal-regulated kinases (ERK) and phosphatidylinositol 3 kinase $(\mathrm{PI} 3 \mathrm{~K}) / \mathrm{Akt}$ pathways ${ }^{[13]}$. cAMP response element-binding protein (CREB), one of the phosphorylated substrates of ERK, is critical for long-term memory formation. The activation of CREB through phosphorylation of Ser133 controls the induction of many genes, including BDNF and Bcl-2 ${ }^{[14,15]}$. In addition, evidence suggests a proapoptotic state in neurons of the AD brain ${ }^{[16]}$. Bcl-2 plays an important role in inhibiting both apoptosis and necrosis of central nervous system cells against several kinds of insults ${ }^{[17]}$. Bcl-2 inhibits cytochrome $c$ release from mitochondria elicited by the proapoptotic molecule Bax, resulting in inhibition of caspase activation and apoptotic death $^{[18,19]}$.

Compound FLZ (chemical name N-[2-(4-hydroxy-phenyl)ethyl]-2-(2,5-dimethoxy-phenyl)-3-(3-methoxy-4-hydroxyphenyl)-acrylamide) is a cyclic analogue of squamosamide found in Annona glabra ${ }^{[20]}$. Our previous studies demonstrated that compound FLZ improved the learning and memory deficit in several experimental animal models of dementia, including APP/PS1 mice ${ }^{[21-23]}$. FLZ exerted most of its neuroprotective effects by reducing $A \beta$ level and aggregation ${ }^{[21]}$ and tau hyperphosphorylation (unpublished data), as well as by protecting mitochondrial function ${ }^{[23-25]}$. Here, we used the APP/ PS1 mouse model, a model that recapitulates many of the salient features of $\mathrm{AD}^{[26]}$, to directly investigate whether modulation of the hippocampal BDNF signaling pathway and/or neuronal apoptosis is involved in FLZ neuroprotection.

\section{Materials and methods} Materials

FLZ is a water-insoluble white powder with $99 \%$ purity. The rabbit anti-active caspase-3 fragment antibody was purchased from Abcam (Cambridge, UK). Rabbit anti-TrkB, anti-p-TrkB (Thr515), and anti-pAkt (Ser473) antibodies were purchased from Signalway Antibody (Pearland, USA). Rabbit antipCREB (Ser133) and anti-CREB antibodies were obtained from Bioworld Technology (MN, USA). Goat anti-Akt1/2 antibody was provided by Boaosen Co (Beijing, China). Mouse antiBcl-2, anti-Bax and rabbit anti-p-ERK, anti-ERK1/2, anti-NGF, anti-BDNF, anti-NT3, and anti-caspase-3 antibodies were products of Santa Cruz Biotechnologies (Heidelberg, Germany).

\section{Animals and FLZ treatment}

The APP/PS1 mice (Tgs) were provided by Prof Lian-feng ZHANG of the Institute of Laboratory Animal Science, Chinese Academy of Medical Science. The animals were gen- erated by co-injection of chimeric mouse/human APP695 harboring the Swedish mutation and human PS1-dE9 vectors, both controlled by their own mouse prion protein promoter element ${ }^{[27]}$. Wild-type (WT) siblings were used as controls. Mice were approximately 4 months old upon their arrival in the laboratory. They were acclimated for 1 month to their new environment before treatments. Nineteen APP/PS1 mice (ten male and nine female mice) and ten WT mice (five male and five female mice) were used in the present study. Nine APP/ PS1 mice (at the age of 5 months) were intragastrically (ig) administered FLZ $(150 \mathrm{mg} / \mathrm{kg}$ in $0.05 \%$ carboxymethyl cellulose sodium (CMC-Na)) daily for 20 weeks. APP/PS1 control and WT control mice were given vehicle $(0.05 \% \mathrm{CMC}-\mathrm{Na})$ ig at the same time. Housing, breeding and experimental use of the animals were performed strictly based on the NIH guidelines for animal care.

\section{Immunohistochemical analysis}

Mice were anesthetized and transcardially perfused with 200 $\mathrm{mL}$ normal sodium (NS) followed by $400 \mathrm{~mL} 4 \%$ paraformaldehyde solution at $24 \mathrm{~h}$ after behavior tests. The brain was post-fixed in the same fixation fluid for another $24 \mathrm{~h}$ at $4{ }^{\circ} \mathrm{C}$. The brain was then sliced coronally into $20 \mu \mathrm{m}$ sections with a Vibratome (Leica, VT1000S, Germany). The frozen sections were permeabilized with $0.3 \% \mathrm{H}_{2} \mathrm{O}_{2}$ (in absolute methanol) for $10 \mathrm{~min}$ to block endogenous peroxidase. Then, the sections were blocked with $5 \%$ BSA for $1 \mathrm{~h}$ at $37^{\circ} \mathrm{C}$ and incubated with BDNF (1:100, Santa Cruz) for $48 \mathrm{~h}$ at $4{ }^{\circ} \mathrm{C}$. After being washed with PBS, sections were subsequently incubated with horseradish peroxidase (HRP)-labeled secondary antibodies for $1 \mathrm{~h}$ at $37^{\circ} \mathrm{C}$. The immunoreactivity was visualized with the diaminobenzidine tetrachloride system. All immunoreactive areas were quantified using ImagePro Plus 6.0 software (Media Cybernetics, Silver Spring, MD). Data were expressed as integrated optical density (IOD), which is equal to the area $\times$ average density of the hippocampus occupied by immunoreactivity and represented as the mean \pm SEM.

\section{Western blotting}

The frozen hippocampal samples were homogenized for 20 min at $4{ }^{\circ} \mathrm{C}$ in a buffer containing $1 \%$ Nonidet NP-40, $1 \%$ Triton-100, 5 mmol/L EDTA, 50 mmol/L Tris-HCl (pH 7.4), $150 \mathrm{mmol} / \mathrm{L} \mathrm{NaCl}$, and a cocktail of protease inhibitors (100×) (Amersco). Extracts were centrifuged at $11430 \times g$ for $20 \mathrm{~min}$ at $4{ }^{\circ} \mathrm{C}$, and the total amount of protein from each sample was determined using the BCA Protein assay kit (Pulilai, Beijing, China). Equal amounts of protein were subjected to SDS-polyacrylamide gel electrophoresis (PAGE) on an 8\% (TrkB), 12\% (BDNF, NT3) or 10\% (Akt, ERK, CREB and caspase-3) acrylamide gel. The resolved proteins were transferred electrophoretically to $0.45 \mu \mathrm{m}$ PVDF membranes (Invitrogen). Membranes were blocked with $5 \%(w / v)$ low-fat milk in $20 \mathrm{mmol} / \mathrm{L}$ Tris, $500 \mathrm{mmol} / \mathrm{L} \mathrm{NaCl}$, and $0.05 \%(w / v)$ Tween-20 ( $\mathrm{pH} 7.5)$ for $1 \mathrm{~h}$. Immunoblotting was performed using antibodies against Bcl-2 (1:1000), Bax (1:1000), active caspase-3 fragment (1:1000), caspase-3 (1:1000), NGF (1:500), BDNF (1:500), NT3 (1:500), 
pAkt1 (Ser473) (1:1000), Akt1/2 (1:1000), pERK (1:1000), ERK (1:1000), pCREB (Ser133) (1:1000) and CREB (1:1000). Membranes were incubated with HRP-conjugated IgG (1:4000), and labeled proteins were detected using the ECL Plus kit (Pulilai Company). The bands were visualized using a Kodak Digital Science ID and quantified with Image Pro Plus 4.0 software. Anti- $\beta$-actin antibody (Santa Cruz) was used as a loading control. All samples were analyzed at least in triplicate.

\section{Statistical analysis}

Statistical analysis was carried out using one-way ANOVA by SPSS 10.0 statistical software. One-way ANOVA was used followed by Dunnett's multiple comparison tests. Data were presented as means \pm SEM, and statistical significance was assumed if $P<0.05$.

\section{Results}

FLZ treatment increased BDNF levels in the hippocampus of APP/PS1 mice

To test the potential neuroprotective effects of FLZ in APP/ PS1 mice, they were treated with FLZ ( $n=9$ mice) at a dose of $150 \mathrm{mg} / \mathrm{kg}$ or $0.05 \%$ CMC-Na ( $n=10$ mice) alone starting at 5 months of age for 20 weeks. The mice were then sacrificed, and the brains were processed for immunohistochemistry ( $n=4$ mice) and western blot analysis ( $n=5$ or 6 mice). The immunohistochemical analysis showed that FLZ treatment of the APP/PS1 group (Figure 1) significantly increased the IOD of the BDNF-immunoreactive neurons $(P<0.05)$. Consistent with the immunostaining result, the immunoblot analysis performed with the anti-BDNF antibody demonstrated that FLZ significantly increased BDNF expression in the hippocampus of APP/PS1 mice $(P<0.05)$.

FLZ treatment promoted TrkB receptor phosphorylation and activated downstream ERK in APP/PS1 mice

To further investigate whether FLZ affected the BDNF/TrkB pathway to exert its neuroprotection, immunoblot analysis was performed using the antibodies against pTrkB (Thr515) and TrkB. This study showed that, compared with WT mice, the pTrkB (Thr515) levels and the pTrkB (Thr515)/TrkB ratio both decreased in the hippocampus of APP/PS1 mice (Tgs) $(P<0.05)$. pTrkB expression and the pTrkB $($ Thr515)/TrkB ratio increased in the brains of the APP/PS1 mice after FLZ $(150 \mathrm{mg} / \mathrm{kg})$ treatment for 20 weeks $(P<0.05)$. However, the levels of TrkB did not change among the three groups $(P>0.05)$ (Figure 2).

To further investigate the mechanism involved in FLZ-mediated neuroprotection, the activation/phosphorylation of the PI3K/Akt and MAPK (MEK/ERK) pathways were studied. In APP/PS1 mice, the ratios of pAkt (Ser473) to Akt $(P<0.05)$ and pERK to ERK $(P<0.05)$ were both decreased notably compared with the WT group. These findings suggested that, compared with the WT groups, Akt and ERK were both inactivated in the hippocampus of the APP/PS1 mice. Strikingly, after FLZ treatment, the APP/PS1 mice showed a remarkable increase in the expression of phosphorylated ERK $(P<0.05)$ but not Akt
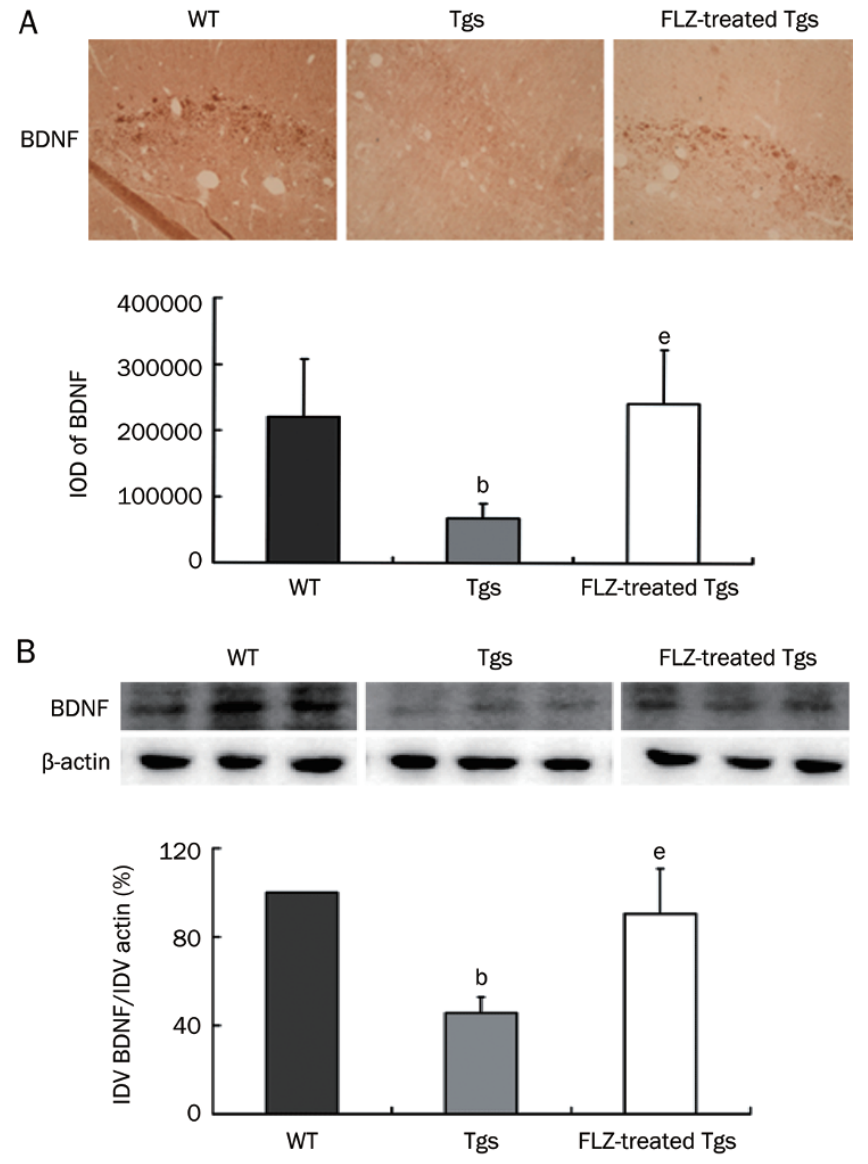

Figure 1. FLZ treatment increased the expression of BDNF in the hippocampus of APP/PS1 mice. Five-month-old mice were treated with vehicle $(0.05 \% \mathrm{CMC}-\mathrm{Na})$ or FLZ $(150 \mathrm{mg} / \mathrm{kg})$ daily for 20 weeks. (A) Representative immunohistochemistry images of BDNF in the hippocampus of wild type (WT) and APP/PS1 (Tgs) mice (4 mice per group). BDNF immunoreactivity was quantification by Image Pro Plus 6.0 software. IOD, integrated optical density. (B) Representative Western blot bands showing BDNF expression in the hippocampus of WT and Tgs mice treated with vehicle or FLZ (150 mg/kg). $n=5-6$ mice per group. Three representative Western blots for each group are shown. The integrated density value was determined using densitometry. IDV, integrated density value. WT, wild-type mice; Tgs, APP/PS1 mice treated with 0.05 CMC-Na; FLZ-treated Tgs, APP/PS1 mice treated with FLZ (150 mg/kg). Results are expressed as means \pm SEM. ${ }^{\mathrm{b}} P<0.05$ vs WT. ${ }^{\mathrm{e}} \mathrm{P}<0.05$ vs Tgs.

$(P>0.05)$. These results suggested that activation of ERK might contribute to the neuroprotection imparted by FLZ treatment (Figure 3).

FLZ treatment increased pCREB (Ser133) expression and the pCREB (Ser133)/CREB ratio in the hippocampus of APP/PS1 mice

An increased $A \beta$ level downregulates CREB phosphorylation, leading to a decrease in BDNF expression ${ }^{[28]}$, we detected the pCREB (Ser133) and CREB expressions by Western blot analysis in order to explore whether FLZ treatment enhanced the BDNF protein level through affecting pCREB (Ser133) level in the mouse hippocampus. The present results have 

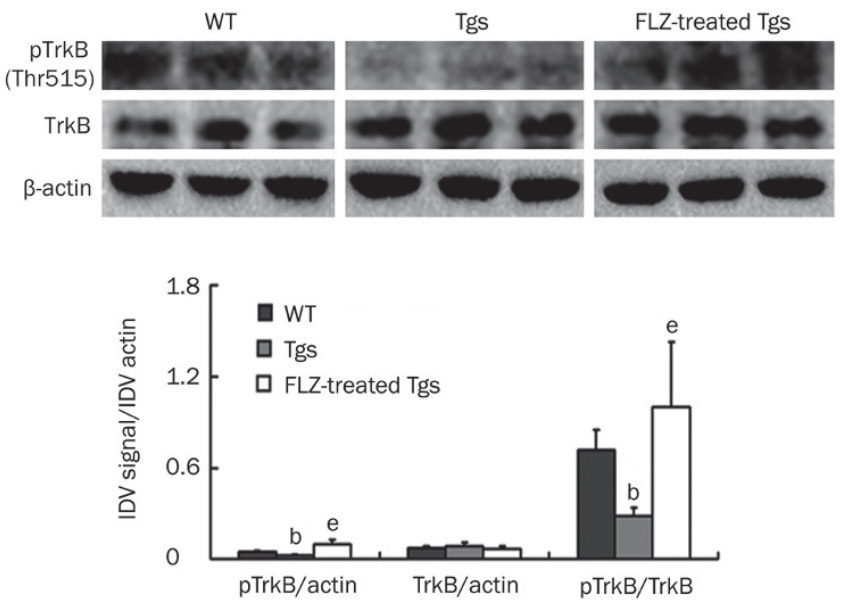

Figure 2. FLZ treatment increased pTrkB (Tyr515) levels and the pTrkB (Tyr515)/TrkB ratio in the hippocampus of APP/PS1 mice. Five-month-old mice were treated with vehicle $(0.05 \% \mathrm{CMC}-\mathrm{Na})$ or FLZ $(150 \mathrm{mg} / \mathrm{kg})$ for 20 weeks. Levels of TrkB and pTrkB (Tyr515) were detected by Western blot and analyzed by Gel Pro Plus 4.0. $n=5-6$ mice per group. Three representative Western blots for each group are shown. IDV, integrated density value. Data are expressed as means \pm SEM. ${ }^{b} P<0.05$ Tgs vs WT. ${ }^{\mathrm{e}} \mathrm{P}<0.05$ FLZ-treated Tgs vs Tgs.

showed that in the hippocampus of APP/PS1 mice, compared with the WT group, pCREB (Ser133) decreased markedly $(P<0.01)$ whereas no change in CREB expression $(P>0.05)$. FLZ increased pCREB (Ser133) expression and the ratio of pCREB (Ser133)/CREB in the hippocampus of APP/PS1 mice $(P<0.05)$, suggesting that FLZ increases BDNF expression by activating CREB (Figure 4).

FLZ treatment increased NT3, but not NGF, expression in the hippocampus of APP/PS1 mice

Because NGF and NT3 play vital roles in neuroplasticity and neuronal fate, we measured NGF and NT3 expressions by Western blot analysis. The data showed that, compared with the WT group, there was no significant change in NGF expression $(P>0.05)$ but a significant decrease in NT3 expression $(P<0.01)$ in the hippocampus of APP/PS1 mice. Compared with the transgenic control group, the FLZ-treated transgenic group had significantly increased NT3 expression $(P<0.01)$ but only a non-significant tendency toward increased NGF expression in the APP/PS1 mouse hippocampus (Figure 5).

\section{FLZ treatment inhibited neuronal apoptosis in the hippocampus of APP/PS1 mice}

Nissl body staining is a marker of mature neurons and can be used to detect neuronal apoptosis. Our Nissl staining analysis showed that, compared with the WT mice, typical neuropathological changes were observed in the CA1 region of hippocampus in APP/PS1 mice, including neuron loss and nucleus shrinkage or disappearance. Furthermore, the numbers of Nissl bodies significantly decreased by $38.15 \%(P<0.05)$ in APP/PS1 mice relative to WT mice. FLZ treatment for 20 weeks significantly decreased the neuropathological changes
A
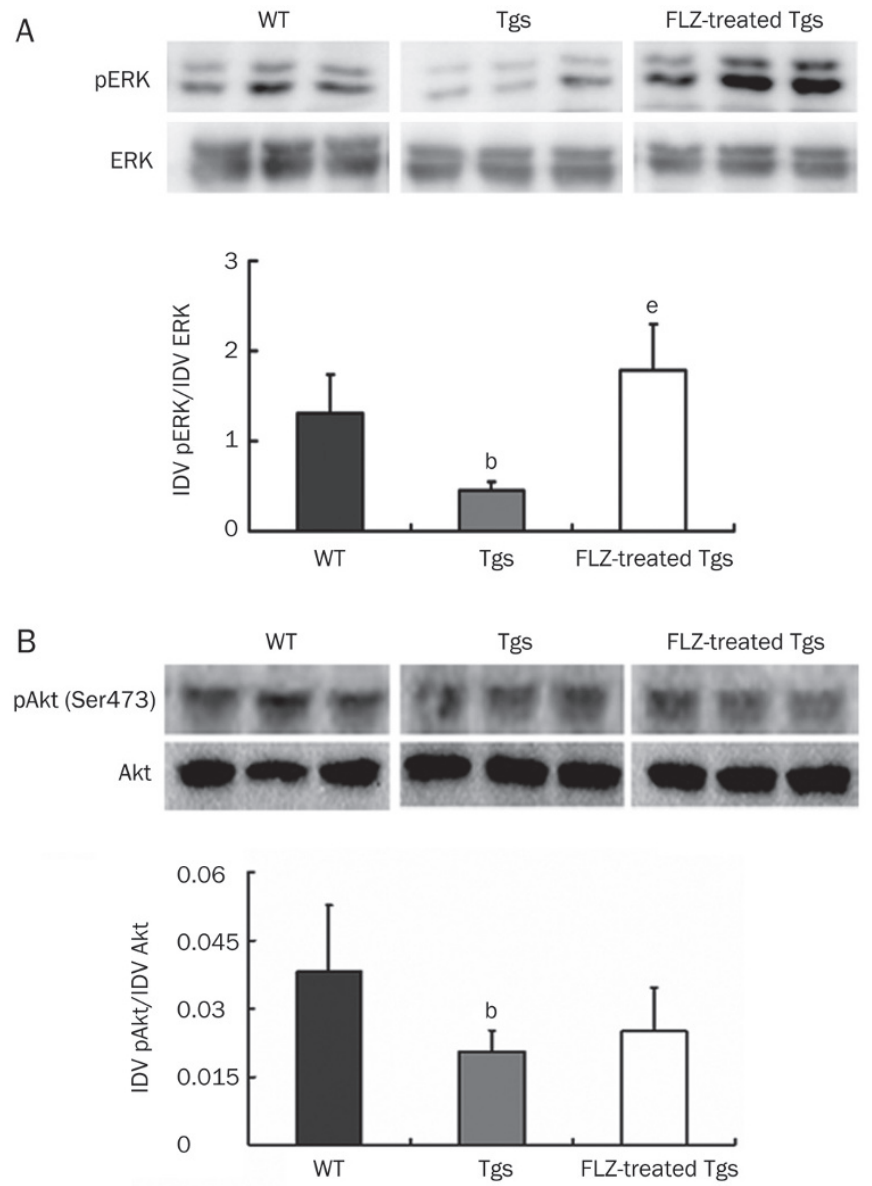

Figure 3. FLZ treatment activated ERK but not Akt in the hippocampus of APP/PS1 mice. Five-month-old mice were treated with vehicle $(0.05 \%$ CMC-Na) or FLZ (150 mg/kg) for 20 weeks. The levels of pERK, total ERK, pAkt (Ser473) and Akt (1/2) were detected by Western blot and analyzed by Gel Pro Plus 4.0. Figure A, Representative western blot bands of pERK and ERK in the hippocampus of WT and Tgs mice. Figure B, Representative Western blot bands of pAkt and Akt in the hippocampus of different groups treated with vehicle or FLZ (150 mg/kg). IDV, integrated density value. $n=5-6$ mice per group. Three representative Western blot for each group are shown. Means \pm SEM. ${ }^{b} P<0.05$ vs WT. ${ }^{e} P<0.05$ vs Tgs.

and increased the density of healthy neurons in the CA1 region in APP/PS1 mice by $34.05 \%(P<0.05)$. These findings indicate that FLZ protected against neuronal apoptosis in the hippocampus of APP/PS1 mice (Figure 6).

FLZ showed potential anti-apoptotic activity by increasing the $\mathrm{Bcl}-2 / \mathrm{Bax}$ ratio and decreasing the active caspase-3 fragment/ caspase-3 ratio in APP/PS1 mice

The expression of apoptotic proteins, including Bcl-2, Bax, active caspase- 3 fragment and caspase- 3 , was detected by Western blot analysis. Compared with the WT group, there was a significant decrease in the $\mathrm{Bcl}-2 / \mathrm{Bax}$ ratio $(P<0.05)$ and a significant increase in the active caspase- 3 fragment/caspase-3 ratio $(P<0.05)$ in APP/PS1 mice. These results are consistent with the result of the Nissl staining, further supporting the evidence of neuronal apoptosis in the hippocampus of APP/ 

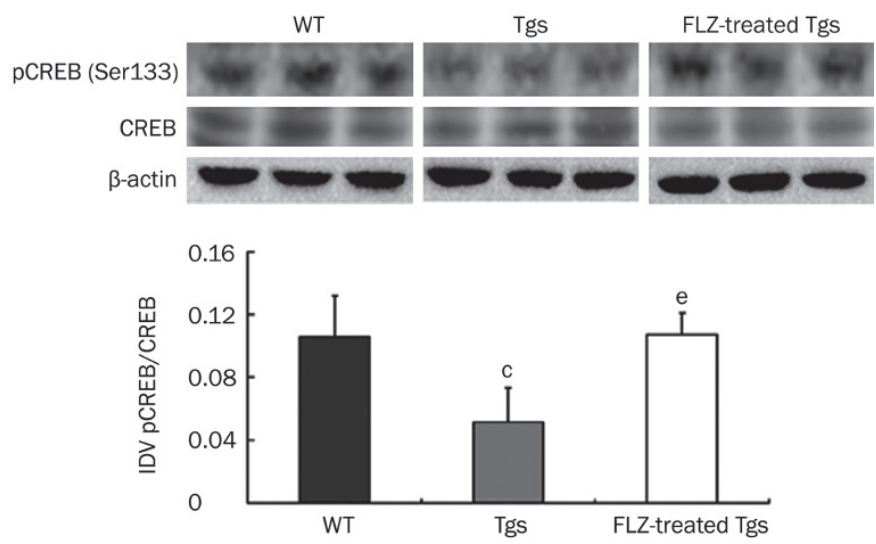

Figure 4. FLZ increased pCREB (Ser133) expression in the hippocampus of APP/PS1 mice. Five-month-old mice were treated with vehicle $(0.05 \%$ CMC-Na) or FLZ (150 mg/kg) for 20 weeks. pCREB (Ser133) levels were detected by Western blot and analyzed by Gel Pro Plus 4.0. $n=5-6$ mice per group. Three representative Western blots for each group are shown. Data are expressed as means \pm SEM. ${ }^{\mathrm{c}} P<0.01$ Tgs vs WT. ${ }^{\mathrm{e}} P<0.05$ FLZtreated Tgs vs Tgs.

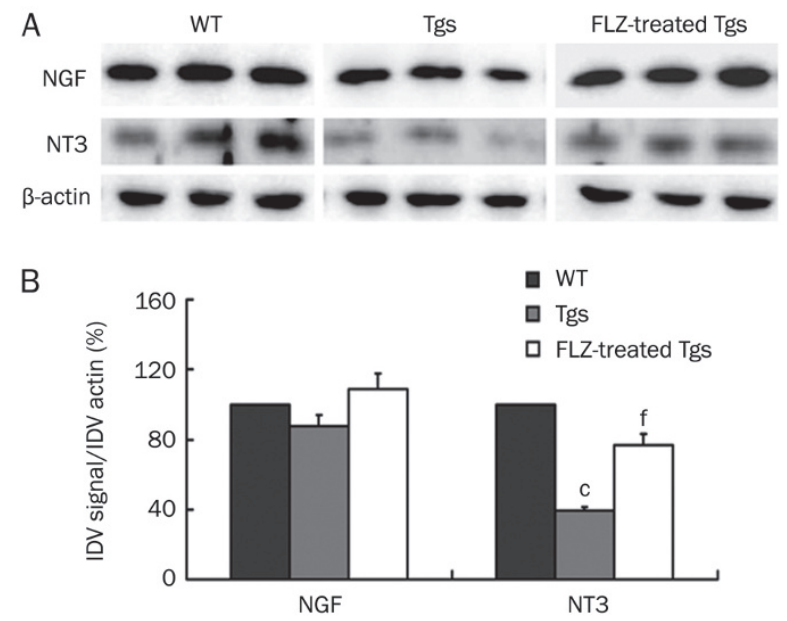

Figure 5. FLZ treatment increased NT3 expression but not NGF expression in the hippocampus of APP/PS1 mice. Five-month-old mice were treated with vehicle $(0.05 \%$ CMC-Na) or FLZ $(150 \mathrm{mg} / \mathrm{kg})$ for 20 weeks. (A) Representative Western blot bands of NGF and NT3 in the hippocampus of WT and Tgs mice. (B) Densitometric quantification of NGF and NT3 expression in the hippocampus of WT and Tgs mice. IDV, integrated density value. $n=5-6$ mice per group. Three representative Western blots for each group are shown. Results are expressed as means \pm SEM. ${ }^{c} P<0.01$ Tgs vs WT. ${ }^{\mathrm{f}} P<0.01$ FLZ-treated Tgs vs Tgs.

PS1 mice. In the FLZ treated group, the expression of Bcl-2 increased $(P<0.05)$ and the level of Bax decreased $(P<0.05)$. In addition, compared with the transgenic group, the Bcl-2/ Bax ratio in the FLZ-treated APP/PS1 group was markedly upregulated $(P<0.05$, Figure $7 \mathrm{~A}$ and $\mathrm{B})$. Finally, FLZ treatment decreased the active caspase- 3 fragment/caspase- 3 ratio in the hippocampus of APP/PS1 mice $(P<0.05$, Figure $7 \mathrm{C}$ and $D)$. These results suggest that FLZ might have potential anti-

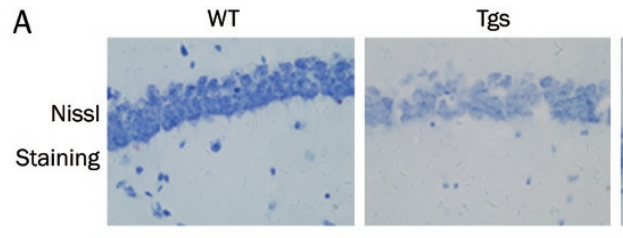

FLZ-treated Tgs

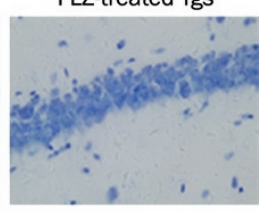

B

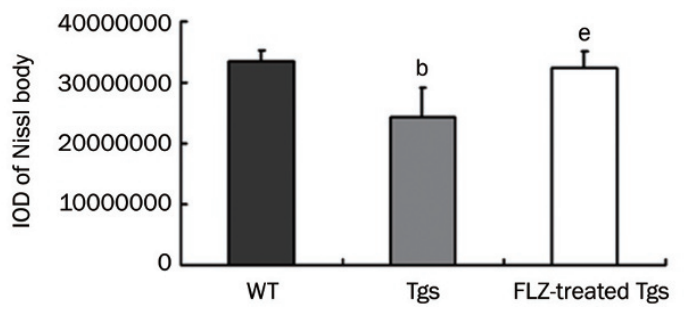

Figure 6. FLZ treatment inhibited neuronal apoptosis in the hippocampus of APP/PS1 mice by Nissl staining. Five-month-old mice were treated with vehicle $(0.05 \% \mathrm{CMC}-\mathrm{Na})$ or FLZ $(150 \mathrm{mg} / \mathrm{kg})$ for 20 weeks. (A) Representative Nissl staining images showing Nissl bodies in the hippocampal CA1 regions of WT and Tgs mice. (B) Quantification of Nissl bodies in the hippocampus of WT and Tgs mice (4 mice per group) by Image Pro Plus 6.0 software. IOD, integrated optical density. ${ }^{\mathrm{b}} P<0.05$, Tgs vs WT. ${ }^{e} P<0.05$, FLZ-treated Tgs vs Tgs.

apoptotic activity on neurons by increasing the Bcl-2/Bax ratio and decreasing the active caspase- 3 fragment/caspase- 3 ratio.

\section{Discussion}

Our previous studies showed that FLZ improved cognitive deficit and reduced $A \beta$ deposits in APP/PS1 mice ${ }^{[21]}$. In this study, the results demonstrated that chronic treatment with FLZ for 20 weeks enhanced BDNF/TrkB/CREB signaling pathway and inhibited neuronal apoptosis in the hippocampus of APP/PS1 mice.

In the present study, APP/PS1 mouse model was used to investigate the beneficial effect of FLZ. Transgenic mouse model, which associated with familial forms of AD, offered a new powerful animal model to study the pathogenesis of $A D$ and to explore the new therapeutic strategy for this disease. Mutations in the APP, presenilin-1, and presenilin-2 genes have been identified as one of the causes of familial forms of $\mathrm{AD}^{[29]}$. All of the three gene mutations have been shown to increase the A $\beta$ burden markedly ${ }^{[30]}$. Mice carrying both APP Swedish mutation and PS1-A246E mutation are developed as a model of AD with more severe pathology than single transgenic for APP or PS1 mutation mice. Such APP/PS1 mice develop large numbers of $A \beta$ deposits in cerebral cortex and hippocampus much earlier than age-matched mice expressing the APP or PS1 mutation alone ${ }^{[31]}$. In view of $A \beta$ deposition is an early and central event in the pathogenesis of $\mathrm{AD}$, the early appearance of $\mathrm{A} \beta$ deposition in APP/PS1 mouse brain developed many other facets of AD neuropathology, such as tau hyperphosphorylation, neuron loss in hippocampus, changes in neurotrophins, deficit in synaptic transmission, changes in behavior and deficits in lone-term potentiation ${ }^{[32]}$.

Neurotrophins, especially BDNF, regulate long-term poten- 
A

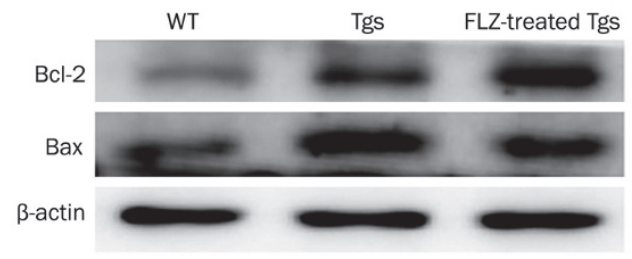

B

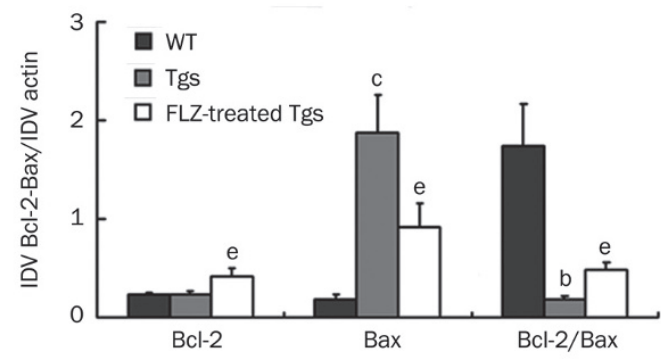

C

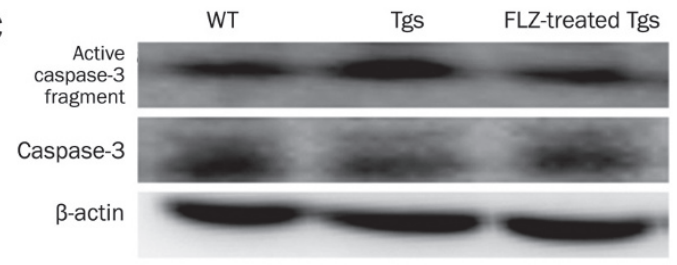

D

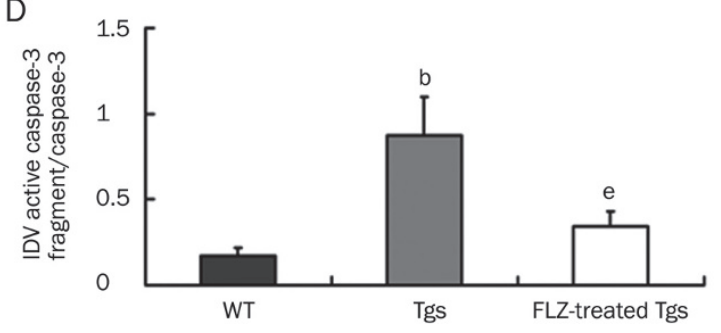

Figure 7. FLZ treatment increased the $\mathrm{Bcl}-2 / \mathrm{Bax}$ ratio and decreased the active caspase-3 fragment/caspase-3 ratio in the hippocampus of APP/ PS1 mice. Five-month-old mice were treated with vehicle $(0.05 \% \mathrm{CMC}$ $\mathrm{Na})$ or FLZ (150 mg/kg) for 20 weeks. (A) Representative Western blot bands of Bcl-2 and Bax in the hippocampus of WT and Tgs mice. (B) Representative Western blot bands of active caspase-3 fragment and caspase- 3 in the hippocampus of different groups. IDV, integrated density value. $n=5-6$ mice per group. Three representative Western blots for each group are shown. Data are expressed as means \pm SEM. ${ }^{\mathrm{b}} P<0.05$, ${ }^{\mathrm{c}} P<0.01$ Tgs vs WT. ${ }^{\mathrm{e}} P<0.05$, FLZ-treated Tgs vs Tgs.

tiation and other types of synaptic plasticity-processes, which have key roles in memory formation and storage. Increased $A \beta$ levels downregulates CREB phosphorylation, leading to a reduction of CRE-dependent gene expression and a decrease in BDNF level followed, and this interferes with induction of synaptic plasticity and memory might contribute to the pathogenesis of $\mathrm{AD}^{[28]}$. In our previous studies, we have shown that FLZ $150 \mathrm{mg} / \mathrm{kg}$ treatment could improve behavior deficit in water maze ${ }^{[21]}$, reduce $A \beta$ production ${ }^{[21]}$, and tau hyperphosphorylation (unpublished data) in APP/PS1 mice. To further explain the mechanisms underlying the beneficial effect of FLZ in APP/PS1 mice, we investigated the potential implication of BDNF. In this study, there was a decrease in BDNF expres- sion in the APP/PS1 mouse hippocampus compared with WT group. We also observed that in the hippocampus of APP/ PS1 mice, TrkB autophosphorylation induced by BDNF was downregulated and its downstream enzymes including Akt and ERK were inactivated, which were detected by the phosphorylation forms of Akt and ERK. We also found a decrease in $\mathrm{pCREB}$ expression activated by ERK. These results indicate that $\mathrm{A} \beta$ deposition can cause dysfunction of the BDNF/TrkB/ CREB signaling pathway. Studies have shown that changes in BDNF and its high-affinity receptor, TrkB, contribute to the loss of synaptic plasticity. It has also been shown that changes in the activity of Akt and ERK are involved in the neuronal apoptosis seen in this AD mouse model ${ }^{[33]}$. FLZ treatment may exert a protective effect by enhancing the expression of BDNF, which, in turn, induces TrkB autophosphorylation, activates ERK and increases CREB phosphorylation in the hippocampus of APP/PS1 mice. The present results support this hypothesis. First, both immunohistochemistry and Western blot results indicated that administration of FLZ for 20 weeks markedly increased the level of BDNF in the hippocampus of APP/PS1 mice. The increased phosphorylation of TrkB at Thr515, through which BDNF exerts its neuronal protective functions coincided with increased expression of BDNF. Correlated with TrkB activation, FLZ treatment activated ERK but not Akt in the hippocampus of APP/PS1 mice. As previously described, activated TrkB interacts with and phosphorylates several intracellular targets, including PI3K/Akt, Ras/MAPK/ ERK, CaM signal pathways ${ }^{[34]}$. Activated Akt phosphorylates its substrates and affects the activities of many kinases, including the apoptotic factors BAD and p53. By comparison, activation of ERK by BDNF mediates mitogenesis, differentiation, and cell survival through phosphorylation of its downstream targets, including Rsks and CREB. CREB activation appears to be a critical step in the signaling cascade that leads to the structural changes underlying the development of long-term memory ${ }^{[35]}$. Interestingly, despite the fact that BDNF can induce the activation of CREB by activating ERK and generate sustained CREB signaling, this CREB signaling loop ultimately involves controlling the induction of $\mathrm{BDNF}^{[36]}$ and there are evidence that $\mathrm{pCREB}$ is a key element in $\mathrm{A} \beta$-induced neurotrophins (BDNF) gene expression reduction ${ }^{[37]}$. The increased CREB phosphorylation by FLZ treatment, in this study, together with its inhibitory effect on $A \beta$ might partly explain the mechanisms of FLZ elevating BDNF expression in APP/ PS1 mice. The above data suggested that FLZ might have the potency to affect neuroplasticity and the long term memory by affecting BDNF/TrkB/CREB signaling pathway.

BDNF has already been as a key molecular target for drug development in neurological disorders. Although there have been some successes, the main problem related to technical details, including a shorter in vivo half-life, a lower bloodbarrier penetrability, and limited diffusion ${ }^{[38,39]}$. Therefore, identification of small molecules that mimic some or all of the properties of neurotrophic factors could have significant potential for treating AD. FLZ is a small molecule that is able to cross the blood brain barrier and is concentrated in the 
brain when administrated systemically (unpublished data). Furthermore, in this study, 20-week administration of FLZ had no adverse effects on mice, including weight loss or locomotor activity changes. All of the findings suggest that FLZ might be a potential neuroprotective agent for $\mathrm{AD}$.

We also observed the effect of FLZ on the other neurotrophins, including NGF and NT3. Regardless of the cause of the cholinergic loss and its contribution to AD, NGF and NT3 levels are diminished in the basal forebrain of $\mathrm{AD}$ patients ${ }^{[40]}$, and many studies have suggested that cognitive performance, neuronal health, and cholinergic activity are improved with NGF treatment in animal models and AD patients ${ }^{[4]}$. In this study, there were a decrease in NT3 expression but had no change in NGF expression in the hippocampus of APP/PS1 mice. Our result did not show a significant improvement of NGF after FLZ treatment. On the contrary, FLZ markedly increased NT3 expression in the hippocampus of APP/PS1 mice. Interestingly, NT3 also exerts its neuroprotective effect on neuronal survival and proliferation by binding and affecting TrkB signal pathway $^{[22,43]}$. These data suggested that FLZ also exerts its neuroprotection through affecting NT3 expression additionally. Although increased pCREB expression partly explains the effect of FLZ on BDNF expression, the concrete mechanism by which FLZ increased BDNF and NT3 expression remains to be elucidated. Therefore, further work will be required.

APP/PS1 mice have been reported to show brain atrophy and substantial cell loss by as early as 6 months of age $\mathrm{e}^{[32]}$. And a severe hippocampal cell loss (50\%) has been reported at the age of 10 months $^{[44]}$. In our study, we found that APP/PS1 mice displayed a significant decrease of hippocampal neurons at the age of 10 months by Nissl staining. Compared with WT mice, there were also markedly changes in the expressions of the apoptotic related proteins, including a decrease in the $\mathrm{Bcl}-2 / \mathrm{Bax}$ ratio and an increase in the active caspase- 3 fragment/caspase-3 ratio in the APP/PS1 mouse hippocampus. Previous studies showed that FLZ attenuated A $\beta 25-$ 35-induced toxicity in SH-SY5Y cells and prevented the hippocampus injury and cognitive deficits induced by A $325-35$ in mice ${ }^{[23]}$. Its anti-apoptotic activity might be related with its regulation of the apoptosis protein (Bcl-2 and Bax) expression and protection of the mitochondrial function ${ }^{[45]}$. In agreement with the previous results, we also found that FLZ treatment inhibited the hippocampal neuronal apoptosis by increasing the ratio of $\mathrm{Bcl}-2 / \mathrm{Bax}$ and decreasing the active caspase-3 fragment markedly in APP/PS1 mice. It is reported that BDNF protects neurons from $A \beta$-induced neuronal apoptosis in vitro and in vivo ${ }^{[12]}$. The Akt and the ERK pathways are two major intracellular signaling network activated by BDNF involved in cell survival. Furthermore, the phosphorylation of CREB by BDNF can also induce Bcl-2 gene transcription ${ }^{[15]}$. The previous studies suggested that increasing Bcl-2 leads to neuronal resistance against apoptotic and oxidative injury ${ }^{[46]}$. The present results suggested that it is possible, that there was a relationship between FLZ-induced BDNF increase and the reduced neuronal apoptosis.

In conclusion, the main finding of this study is that FLZ enhanced BDNF/TrkB/CREB signaling pathway and inhibited neuronal apoptosis. Such effects might partly explain the persistent improvement in cognitive functions in APP/ PS1 mouse model following chronic FLZ treatment. Because $\mathrm{AD}$ is a multifactorial disease with complicated pathogenesis, exploring the multi-targets drugs might be an innovative and perspective therapy strategy ${ }^{[47]}$. Considering that FLZ showed several potentially beneficial effects on neurodegeneration processes, together with the ability to decrease $A \beta$ production $^{[21]}$, to cross the blood brain barrier and its lower toxicity, FLZ may be explored as a potential therapeutic agent in long-term $\mathrm{AD}$ therapy.

\section{Abbreviations}

$\mathrm{AD}$, Alzheimer's Disease; APP, the Amyloid precursor protein; $\mathrm{BDNF}$, brain-derived neurotrophic factor; CREB, CAMP response element-binding protein; ERK, extracellular signal-regulated kinases; FDA, Food and Drug Administration; MAPK, mitogenactivated protein kinases; NGF, nerve growth factor; NIH, National Institutes of Health; NT3, neurotrophin 3; PS1, presenilin 1 ; TrkB, tropomyosin receptor kinase B.

\section{Acknowledgements}

Thanks for the kindly suggestions of Prof Lian-feng ZHANG, PhD Dan ZHANG and Prof Jian-jun ZHANG on this work.

\section{Author contribution}

Prof Geng-tao LIU designed the research; Ning LI performed the research and analyzed the data; Ning LI and Prof Geng-tao LIU prepared the manuscript.

\section{References}

1 Scinto LF, Wu CK, Firla KM, Daffner KR, Saroff D, Geula C. Focal pathology in the Edinger-Westphal nucleus explains pupillary hypersensitivity in Alzheimer's disease. Acta Neuropathol 1999; 97: 557-64.

2 Auld DS, Kornecook TJ, Bastianetto S, Quirion R. Alzheimer's disease and the basal forebrain cholinergic system: relations to beta-amyloid peptides, cognition, and treatment strategies. Prog Neurobiol 2002; 68: 209-45.

3 Schliebs R, Arendt T. The significance of the cholinergic system in the brain during aging and in Alzheimer's disease. J Neural Transm 2006; 113: 1625-44.

4 Coleman PD, Yao PJ. Synaptic slaughter in Alzheimer's disease. Neurobiol Aging 2003; 24: 1023-7.

5 Okamura N, Suemoto T, Shiomitsu T, Suzuki M, Shimadzu H, Akatsu $\mathrm{H}$, et al. A novel imaging probe for in vivo detection of neuritic and diffuse amyloid plaques in the brain. J Mol Neurosci 2004; 24: 247-55.

6 Muresan Z, Muresan V. Neuritic deposits of amyloid-beta peptide in a subpopulation of central nervous system-derived neuronal cells. Mol Cell Biol 2006; 26: 4982-97.

7 Sevilla C, Jimenez Caballero PE, Alfonso V, Gonzalez-Adalid M. Current treatments of Alzheimer disease: are main caregivers satisfied with the drug treatments received by their patients? Dement Geriatr Cogn Disord 2009; 28: 196-205.

8 Ockel M, Lewin GR, Barde YA. In vivo effects of neurotrophin-3 during sensory neurogenesis. Development 1996; 122: 301-7.

9 Counts SE, Mufson EJ. The role of nerve growth factor receptors in 
cholinergic basal forebrain degeneration in prodromal Alzheimer disease. J Neuropathol Exp Neurol 2005; 64: 263-72.

10 Tapia-Arancibia L, Aliaga E, Silhol M, Arancibia S. New insights into brain BDNF function in normal aging and Alzheimer disease. Brain Res Rev 2008; 59: 201-20.

11 Fumagalli F, Racagni G, Riva MA. The expanding role of BDNF: a therapeutic target for Alzheimer's disease? Pharmacogenomics J 2006; 6: 8-15.

12 Arancibia S, Silhol M, Mouliere F, Meffre J, Hollinger I, Maurice T, et al. Protective effect of BDNF against beta-amyloid induced neurotoxicity in vitro and in vivo in rats. Neurobiol Dis 2008; 31: 316-26.

13 Murer MG, Yan Q, Raisman-Vozari R. Brain-derived neurotrophic factor in the control human brain, and in Alzheimer's disease and Parkinson's disease. Prog Neurobiol 2001; 63: 71-124.

14 Tao X, Finkbeiner S, Arnold DB, Shaywitz AJ, Greenberg ME. $\mathrm{Ca}^{2+}$ influx regulates BDNF transcription by a CREB family transcription factor-dependent mechanism. Neuron 1998; 20: 709-26.

15 Lonze BE, Ginty DD. Function and regulation of CREB family transcription factors in the nervous system. Neuron 2002; 35 : 605-23.

16 Anderson AJ, Pike CJ, Cotman CW. Differential induction of immediate early gene proteins in cultured neurons by beta-amyloid (A beta): association of c-Jun with A beta-induced apoptosis. J Neurochem 1995; 65: 1487-98.

17 Kane DJ, Sarafian TA, Anton R, Hahn H, Gralla EB, Valentine JS, et al. Bcl-2 inhibition of neural death: decreased generation of reactive oxygen species. Science 1993; 262: 1274-7.

18 Shimizu S, Narita M, Tsujimoto Y. Bcl-2 family proteins regulate the release of apoptogenic cytochrome $c$ by the mitochondrial channel VDAC. Nature 1999; 399: 483-7.

19 Kluck RM, Bossy-Wetzel E, Green DR, Newmeyer DD. The release of cytochrome $c$ from mitochondria: a primary site for $\mathrm{Bcl}-2$ regulation of apoptosis. Science 1997; 275: 1132-6.

20 Xie P, Jiao XZ, Liang XT, Feng WH, Wei HL, Liu GT. Synthesis and antioxiactivity of squamosamide cyclic analogs. Zhongguo Yi Xue Ke Xue Yuan Xue Bao 2004; 26: 372-8.

21 Hou Y, Yu YB, Liu G, Luo Y. A natural squamosamide derivative FLZ reduces amyloid-beta production by increasing non-amyloidogenic AbetaPP processing. J Alzheimers Dis 2009; 18: 153-65.

22 Fang F, Liu G. A novel cyclic squamosamide analogue compound FLZ improves memory impairment in artificial senescence mice induced by chronic injection of $D$-galactose and $\mathrm{NaNO}_{2}$. Basic Clin Pharmacol Toxicol 2007; 101: 447-54.

23 Fang F, Liu GT. Protective effects of compound FLZ on beta-amyloid peptide-(25-35)-induced mouse hippocampal injury and learning and memory impairment. Acta Pharmacol Sin 2006; 27: 651-8.

24 Zhang D, Zhang JJ, Liu GT. The novel squamosamide derivative FLZ protects against 6-hydroxydopamine-induced apoptosis through inhibition of related signal transduction in SH-SY5Y cells. Eur J Pharmacol 2007; 561: 1-6.

25 Zhang D, Zhang JJ, Liu GT. The novel squamosamide derivative (compound FLZ) attenuated 1-methyl, 4-phenyl-pyridinium ion ( $\mathrm{MPP}^{+}$)induced apoptosis and alternations of related signal transduction in SH-SY5Y cells. Neuropharmacology 2007; 52: 423-9.

26 Lamb BT, Bardel KA, Kulnane LS, Anderson JJ, Holtz G, Wagner SL, et al. Amyloid production and deposition in mutant amyloid precursor protein and presenilin-1 yeast artificial chromosome transgenic mice. Nat Neurosci 1999; 2: 695-7.

27 Jankowsky JL, Fadale DJ, Anderson J, Xu GM, Gonzales V, Jenkins NA, et al. Mutant presenilins specifically elevate the levels of the 42 residue beta-amyloid peptide in vivo: evidence for augmentation of a 42-specific gamma secretase. Hum Mol Genet 2004; 13: 159-70.

28 Arancio O, Chao MV. Neurotrophins, synaptic plasticity and dementia. Curr Opin Neurobiol 2007; 17: 325-30.

29 Bertram L, Tanzi RE. Thirty years of Alzheimer's disease genetics: the implications of systematic meta-analyses. Nat Rev Neurosci 2008; 9: 768-78.

30 Selkoe DJ. Alzheimer's disease: genotypes, phenotypes, and treatments. Science 1997; 275: 630-1.

31 Kurt MA, Davies DC, Kidd M, Duff K, Rolph SC, Jennings KH, et al. Neurodegenerative changes associated with beta-amyloid deposition in the brains of mice carrying mutant amyloid precursor protein and mutant presenilin-1 transgenes. Exp Neurol 2001; 171: 59-71.

32 Faure A, Verret L, Bozon B, El Tannir El Tayara N, Ly M, et al. Impaired neurogenesis, neuronal loss, and brain functional deficits in the APPXPS1-Ki mouse model of Alzheimer's disease. Neurobiol Aging 2009 Apr 22. [Epub ahead of print].

33 Marshall CJ. Specificity of receptor tyrosine kinase signaling: transient versus sustained extracellular signal-regulated kinase activation. Cell 1995; 80: 179-85.

34 Segal RA, Greenberg ME. Intracellular signaling pathways activated by neurotrophic factors. Annu Rev Neurosci 1996; 19: 463-89.

35 Bourtchuladze R, Frenguelli B, Blendy J, Cioffi D, Schutz G, Silva AJ. Deficient long-term memory in mice with a targeted mutation of the cAMP-responsive element-binding protein. Cell 1994; 79: 59-68.

36 Bramham CR, Messaoudi E. BDNF function in adult synaptic plasticity: the synaptic consolidation hypothesis. Prog Neurobiol 2005; 76: 99-125.

37 Ikezu T, Okamoto T, Komatsuzaki K, Matsui T, Martyn JA, Nishimoto I. Negative transactivation of cAMP response element by familial Alzheimer's mutants of APP. EMBO J 1996; 15: 2468-75.

38 Levy YS, Gilgun-Sherki Y, Melamed E, Offen D. Therapeutic potential of neurotrophic factors in neurodegenerative diseases. BioDrugs 2005; 19: 97-127.

39 Fumagalli F, Racagni G, Riva MA. Shedding light into the role of BDNF in the pharmacotherapy of Parkinson's disease. Pharmacogenomics J 2006; 6: 95-104.

40 Schindowski K, Belarbi K, Buee L. Neurotrophic factors in Alzheimer's disease: role of axonal transport. Genes Brain Behav 2008; 7 Suppl 1: 43-56.

41 Williams BJ, Eriksdotter-Jonhagen M, Granholm AC. Nerve growth factor in treatment and pathogenesis of Alzheimer's disease. Prog Neurobiol 2006; 80: 114-28.

42 Glass DJ, Nye SH, Hantzopoulos P, Macchi MJ, Squinto SP, Goldfarb M, et al. TrkB mediates BDNF/NT-3-dependent survival and proliferation in fibroblasts lacking the low affinity NGF receptor. Cell 1991; 66: 405-13.

43 Klein R, Nanduri V, Jing SA, Lamballe F, Tapley P, Bryant S, et al. The trkB tyrosine protein kinase is a receptor for brain-derived neurotrophic factor and neurotrophin-3. Cell 1991; 66: 395-403.

44 Casas C, Sergeant N, Itier JM, Blanchard V, Wirths O, van der Kolk N, et al. Massive CA1/2 neuronal loss with intraneuronal and $\mathrm{N}$-terminal truncated Abeta42 accumulation in a novel Alzheimer transgenic model. Am J Pathol 2004; 165: 1289-300.

45 Fang F, Liu GT. Novel squamosamide derivative (compound FLZ) attenuates Abeta25-35-induced toxicity in SH-SY5Y cells. Acta Pharmacol Sin 2008; 29: 152-60.

46 Merry DE, Korsmeyer SJ. Bcl-2 gene family in the nervous system. Annu Rev Neurosci 1997; 20: 245-67.

47 Youdim MB, Buccafusco JJ. CNS targets for multi-functional drugs in the treatment of Alzheimer's and Parkinson's diseases. J Neural Transm 2005; 112: 519-37. 